\title{
Screening for Bipolar Disorder in Patients Treated for Depression in a Family Medicine Clinic
}

\author{
Robert M. A. Hirschfeld, MD, Alvah R. Cass, MD, SM, Devin C. L. Holt, MS, and \\ Carol A. Carlson, BA
}

Purpose: The prevalence of bipolar disorder in patients being treated for depression with antidepressants in a family medicine clinic was investigated in this study.

Methods: Adult patients with depression who were treated with an antidepressant in a general outpatient family medicine clinic at the University of Texas were screened for bipolar disorder using the Mood Disorder Questionnaire (MDQ), a brief self-report inventory. A subsample of subjects received the Structured Clinical Interview based on the DSM-IV (SCID) to test the operating characteristics of the MDQ in this population.

Results: of the patients taking an antidepressant for depression, $21.3 \%$ screened positive for bipolar disorder on the MDQ. These patients were somewhat younger, more likely to be white, more likely to be living alone, much less likely to be married, and less likely to be employed than those who screen negative. Nearly two thirds of those screened positive had never received diagnosis of bipolar disorder. The sensitivity and specificity of the MDQ in this population, after adjusting for the sampling protocol, were $0.580(0.454$ to $0.706,95 \% \mathrm{CI})$ and $0.930(0.878$ to $0.981,95 \% \mathrm{CI})$ respectively.

Conclusion: Bipolar disorder frequently occurs in patients being treated with antidepressants in primary care settings. Most are unrecognized and undiagnosed. Screening for bipolar disorder in such patients may improve recognition, identification, and appropriate treatment. ( $\mathrm{J}$ Am Board Fam Pract 2005;18:233-9.)

The recognition, diagnosis, and treatment of depression in primary care settings has received enormous attention over the last decade. A result of this attention is the management of depressed patients in primary care settings has been improved. Unfortunately, little attention has been given to a significant subset of depressed patients-those with bi-

Submitted, revised, 16 March 2005.

From the Departments of Psychiatry and Behavioral Sciences (RMAH, DCLH), and Family Medicine (ARC, CAC), University of Texas Medical Branch at Galveston, TX.

Funding: This study was supported by a Distinguished Investigator Award from the National Alliance for Research on Schizophrenia and Depression Foundation.

Conflict of interest: RMAH has received active grants or research support from Wyeth-Ayerst; is a consultant to or is on the advisory board of Abbott Laboratories, AstraZeneca, Bristol-Myers Squibb, Forest Laboratories, GlaxoSmithKline, Janssen Pharmaceutica, Eli Lilly \& Company, Novartis, Organon, Inc., Pfizer, Inc., Shire, UCB Pharma, and Wyeth-Ayerst. ARC, CAC, and DCLH have no potential conflicts of interest.

Corresponding author: Robert M. A. Hirschfeld, MD, Department of Psychiatry and Behavioral Sciences, University of Texas Medical Branch, 1.302 Rebecca Sealy, 301 University Boulevard, Galveston, TX 77555-0188 (e-mail: rohirsch@utmb.edu). polar disorder. A notable exception has been the work of J. Sloan Manning in Tennessee, who reported that $26 \%$ of patients presenting with depression in his family practice setting had bipolar disorder. ${ }^{1}$ This prevalence is similar to that reported in the psychiatric specialty care settings. In a study conducted in France by Hantouche et al, ${ }^{2}$ the rate of bipolar disorder in a population of patients presenting with a major depressive episode was $28 \%$. In a study conducted in a private psychiatric practice in northern Italy, Benazzi ${ }^{3}$ found that $49 \%$ of the outpatients presenting with depression had bipolar spectrum disorder. The prevalence of bipolar disorder in these clinical settings is substantially higher than that found in the community, which ranges from approximately $1 \%$ for bipolar I disorder to $3 \%$ to $6.5 \%$ for bipolar I and II and other disorders in the bipolar spectrum. ${ }^{4}$

The issue of misdiagnosis or inaccurate diagnosis is particularly important for patients who are being treated with antidepressants for depression. Antidepressants, particularly tricyclics and monoamine oxidase inhibitors, when administered with- 
out a concurrent mood stabilizer, can destabilize bipolar depression and precipitate a manic episode. ${ }^{5}$ Furthermore, misdiagnosis will prevent the patient with bipolar disorder from receiving medications, such as lithium or divalproex, that will likely treat the disorder. Therefore, it is critical that bipolar patients be identified among depressed patients so appropriate treatment can be administered. This article addresses the frequency of bipolar disorder in a sample of patients who are being treated for depression with antidepressants in a primary care clinic at the University of Texas Medical Branch (UTMB) in Galveston.

\section{Methods}

\section{Sample}

Nearly 9000 unique patients are seen in the Family Medicine Clinic each year by family medicine faculty, residents, physician assistants, and behavioral therapists. Approximately $9.4 \%$ of these patients are diagnosed with depression. Subjects 18 years of age and older taking antidepressants for depression as diagnosed by a family medicine physician or psychiatrist were recruited from an outpatient Family Medicine Clinic at the UTMB at Galveston. Participants were excluded from the study if they discontinued taking the antidepressants because they were not currently suffering from depression or if the antidepressants were not being prescribed for depression. An effort was made to identify all patients who either received a new prescription for an antidepressant medication to treat depression or were currently taking an antidepressant for treatment of depression at the time of any clinic visit. All patients so identified were approached and asked to complete the Mood Disorder Questionnaire (MDQ) and a demographic and clinical history form on a completely voluntary basis. A subsequent sample of people who completed this battery and agreed to participate in an in-depth telephone interview underwent a modified Structured Clinical Interview based on the DSM IV (SCID) within several weeks of completing the MDQ. We attempted to interview all patients who agreed to the interview and who screened positive on the MDQ. We interviewed approximately an equal number of patients sampled from the patients who screened negative on the MDQ and who agreed to the telephone interview. This technique has been used successfully by our research group in other studies. ${ }^{6-8}$ The interviewers for the SCID were all experienced raters and each had completed over 100 SCID interviews before the study. Whenever a patient received a diagnosis of bipolar disorder based on the SCID interview, the primary care physician was notified. The SCID raters were blinded to the results of the MDQ, which was determined by a computer scoring algorithm that maintained blinding during the analysis. Written informed consent was obtained from all participants, and all procedures used in this study were reviewed and approved by the UTMB Institutional Review Board.

\section{Instruments}

The demographic and clinical history form contained general demographic items, including sex, race, education, marital status, etc. The clinical history portion of the form retrieves information about when the patient began taking antidepressants, what they were prescribed for, and whether or not they had been diagnosed previously with bipolar disorder.

The MDQ is a self-report screening inventory for bipolar I and II disorder. The MDQ is a screening instrument and not a diagnostic tool. The MDQ has been validated in a psychiatric outpatient setting and in the general population. ${ }^{7,8}$ The MDQ contains 13 yes/no items, asking about mood, selfconfidence, energy, sociability, interest in sex, and other behaviors and two additional questions that inquire about co-occurrence of symptoms during the same period of time (yes/no question) and about the severity of functional impairment caused by the symptoms on a 4-point scale from "no problem" to "serious problem."7 A positive MDQ screen is defined as endorsement of at least 7 of 13 symptom items, co-occurrence of 2 or more symptoms, and moderate to severe impairment.

The SCID is a structured clinical interview for psychiatric disorders administered by a trained interviewer. ${ }^{9}$ The SCID is widely used in psychiatric clinical research and is generally regarded as the standard for psychiatric diagnosis. ${ }^{10,11}$ An abbreviated lifetime version of the SCID for Axis I disorders was used in this study. ${ }^{8}$ Only the modules assessing major depressive disorder, bipolar spectrum disorders, and alcohol/substance abuse were administered. 


\section{Data Analysis}

The specific aims of the analyses were to estimate the prevalence of bipolar spectrum disorders in a primary care setting in patients taking antidepressants for depression and to determine the operating characteristics of the MDQ in a primary care setting. Operating characteristics of the MDQ were calculated from $2 \times 2$ contingency tables and reported as the crude estimates of sensitivity and specificity. We also report sensitivity and specificity, adjusted for sampling, after we confirmed that the participants who completed the SCID interview were similar to participants who did not complete the SCID. We estimated the $95 \%$ confidence intervals for a binomial proportion using the normal approximation method. An estimate of area under the curve for a receiver operator characteristic curve was calculated using the methods described by Cantor and Katten ${ }^{12}$ for continuous data that are simplified to a dichotomous test.

Sociodemographic data and other parameters were compared using a Fischer exact test or Pear- son $\chi^{2}$ for nominal and ordinal data. Continuous data were compared using a Student $t$ test for independent samples. We used SPSS Version 11.5 (SPSS Inc., Chicago, IL) to conduct all statistical analyses.

\section{Results}

We enrolled 649 primary care patients receiving treatment for depression between April 2003 and March 2004. The mean age of participants was $51 \pm 13$ years (Table 1 ). Women comprised $82 \%$ of the study sample; $72 \%$ of the enrollees were non-Hispanic white; $16 \%$ were African American; $9 \%$ were Hispanic, and $85 \%$ of the subjects had at least a high school education. Married persons made up $42 \%$ of the subjects; $49 \%$ were employed either full or part time; and $73 \%$ lived with a spouse, with a spouse and children, or in an extended family unit. One hundred and five patients (16\%) gave a history of a previous diagnosis of bipolar disorder.

Table 1. Sociodemographic Characteristics versus MDQ Results

\begin{tabular}{|c|c|c|c|c|}
\hline Characteristics & $\begin{array}{c}\% M D Q \text { Positive } \\
\mathrm{N}=138\end{array}$ & $\begin{array}{c}\% M D Q \text { Negative } \\
\mathrm{N}=511\end{array}$ & $\begin{array}{c}\text { \% Total Sample } \\
\mathrm{N}=649\end{array}$ & $P$ \\
\hline \multicolumn{5}{|l|}{ Age } \\
\hline Mean (years) & $47.71 \pm 11.48$ & $51.84 \pm 13.49$ & 50.0 & .005 \\
\hline \multicolumn{5}{|l|}{ Gender } \\
\hline Male & 15.2 & 19.2 & 18.3 & .268 \\
\hline Female & 84.8 & 80.8 & 81.7 & \\
\hline \multicolumn{5}{|l|}{ Racial/ethnic background } \\
\hline White & 76.1 & 70.6 & 71.8 & \\
\hline Black or African American & 15.2 & 16.4 & 16.2 & .017 \\
\hline Hispanic or Mexican American & 2.9 & 10.2 & 8.6 & \\
\hline Other & 5.8 & 2.7 & 3.4 & \\
\hline \multicolumn{5}{|l|}{ Education level } \\
\hline Not a high school graduate & 13.2 & 15.9 & 15.3 & .448 \\
\hline High school graduate or higher & 86.8 & 84.1 & 84.7 & \\
\hline \multicolumn{5}{|l|}{ Family } \\
\hline Alone & 29 & 25.9 & 26.6 & \\
\hline Spouse & 20.3 & 29.7 & 27.7 & .018 \\
\hline Simple & 30.4 & 32.8 & 32.3 & \\
\hline Extended & 20.3 & 11.6 & 13.4 & \\
\hline \multicolumn{5}{|l|}{ Martial Status } \\
\hline Married & 29.7 & 45.4 & 42.1 & \\
\hline Divorced/separated & 45.6 & 28.6 & 35.3 & .001 \\
\hline Widowed & 8 & 12.7 & 11.7 & \\
\hline Single/never married & 16.7 & 13.3 & 13.3 & \\
\hline \multicolumn{5}{|l|}{ Employment status } \\
\hline Employed & 55.1 & 46.8 & 48.5 & \\
\hline Not employed & 33.3 & 31.1 & 31.6 & .021 \\
\hline Retired & 11.6 & 22.1 & 19.9 & \\
\hline \multicolumn{5}{|l|}{ Previous history of bipolar } \\
\hline Yes & 34.8 & 11.2 & 16.2 & .000 \\
\hline No & 65.2 & 88.8 & 83.8 & \\
\hline
\end{tabular}

MDQ, Mood Disorder Questionnaire. 
The endorsement rate of MDQ items in the entire sample ranged from $20 \%$ for "more active. . . than usual" to $68 \%$ for "easily distracted... had trouble concentrating or staying on track" (Table 2 ). Respondents reported clustering of 2 or more symptoms $62 \%$ of the time and indicated that the symptoms produced moderate to serious problems $46 \%$ of the time. The internal reliability of the MDQ in this sample was very good. The Cronbach $\alpha$ coefficients for the 13 -item symptom scale and 15 -item survey were 0.82 and 0.81 , respectively.

Of the 649 patients completing the MDQ, 485 consented to the SCID interview. One hundred eighty patients were selected from the MDQ-positive and the MDQ-negative groups to receive the SCID interview. We found no significant difference in sociodemographic variables when we compared participants who completed the SCID with those who did not (Table 3).

Of the MDQ-positive subjects, 86 of 138 completed a SCID interview, and 94 of 511 of the MDQ-negative subjects completed an interview (Table 4). The MDQ correctly identified 45 of 59 patients diagnosed with bipolar disorder, yielding an unadjusted sensitivity of 0.763 (95\% CI, 0.654 to 0.871 ). The MDQ correctly identified 80 of 121 patients without bipolar disorder, yielding an unadjusted specificity of 0.661 (95\% CI, 0.577 to 0.745). After adjusting for sample, the sensitivity and specificity were 0.580 (95\% CI, 0.454 to 0.706 ,

Table 2. Endorsement of Symptoms in the Entire Sample $(\mathrm{N}=649)$

\begin{tabular}{lcc}
\hline & $\begin{array}{c}\text { Frequency } \\
\text { of Positive } \\
\text { Response }\end{array}$ & $\begin{array}{c}\text { Percentage } \\
\text { of Positive } \\
\text { Response }\end{array}$ \\
\hline Felt so good or so hyper... & 198 & 30.6 \\
Were so irritable... & 408 & 62.9 \\
Felt much more self confident... & 291 & 45.3 \\
Got much less sleep... didn't miss it & 230 & 35.5 \\
Were much more talkative... & 269 & 41.6 \\
Thoughts raced... & 397 & 61.5 \\
Were easily distracted... & 443 & 68.4 \\
Had much more energy... & 215 & 33.1 \\
Were much more active... & 265 & 41.1 \\
Were much more social... & 128 & 19.8 \\
Were much more interested in sex... & 160 & 24.8 \\
Did things that were unusual... & 206 & 32.0 \\
Spending money... & 200 & 30.8 \\
Co-occurrence of symptoms & 384 & 62.0 \\
Symptom severity-moderate/serious & 290 & 45.7 \\
\hline
\end{tabular}

95\%) and 0.930 (95\% CI, 0.878 to 0.981 ), respectively. Using Cantor's ${ }^{11}$ method and assuming a cutoff point of 7 or more symptoms, the area under the curve for the MDQ conditioned on co-occurrence and severity of symptoms was 0.755 (sensitivity, 0.580; specificity, 0.930). Table 5 compares the adjusted operating characteristics of the MDQ from the current study with previous studies conducted in the general population and an outpatient psychiatric population.

The unadjusted sensitivity for detecting bipolar I was 0.775 (95\% CI, 0.646 to 0.904 ); ie, 31 of the 40 SCID-diagnosed bipolar I subjects were correctly identified by the MDQ. The unadjusted sensitivity for detecting bipolar II in patients was 0.706 (95\% CI, 0.445 to 0.879 , exact method); ie, 12 of 17 SCID-diagnosed bipolar II patients were correctly identified by the MDQ. Two patients were classified as bipolar disorder not otherwise specified (NOS).

Of the primary care patients who had been prescribed an antidepressant for depression, $21.3 \%$ (138 of 649) screened positive for bipolar disorder on the MDQ. The MDQ-positive patients were younger (Table 1), more likely to be white, and more likely to be living alone. MDQ-positive subjects were less likely to be married and less likely to be employed. There was no difference in gender between the groups. Subjects who screened positive on the MDQ were more likely to have received a previous diagnosis of bipolar disorder than those who screened negative; however, only one third of the MDQ-positive patients (34.8\%) had been diagnosed previously. Using the distribution of MDQ scores and the results of the 180 completed SCID interviews; we could impute the probability of bipolar disorder in the remaining subjects who did not complete the SCID. This approach provided an estimated prevalence of bipolar disorders of $27.9 \%$ in this sample of primary care subjects currently diagnosed and treated for depression.

Of the entire sample of patients taking antidepressants, $16.2 \%$ (105 of 649) reported a prior diagnosis of bipolar disorder. The SCID interview was conducted on 43 of the 105 people reporting a prior diagnosis of bipolar disorder. The SCID confirmed this history in only $67.4 \%$ (29 of 43 ) of the people (Table 6). Subjects with bipolar I (23 of 40, $57.5 \%$ ) were more likely to report a previous history of bipolar disorder than subjects with bipolar II (6 of 17, 35.3\%). Neither of the subjects with 
Table 3. Comparison of Subjects Who Completed SCID versus Those Who Did Not

\begin{tabular}{|c|c|c|c|c|c|c|c|c|}
\hline \multirow[b]{2}{*}{ Variable } & \multicolumn{4}{|c|}{ MDQ Positive $\mathrm{N}=138$} & \multicolumn{4}{|c|}{ MDQ Negative $\mathrm{N}=511$} \\
\hline & $\begin{array}{c}\text { SCID } \\
\mathrm{N}=83\end{array}$ & $\begin{array}{l}\text { No SCID } \\
\mathrm{N}=54\end{array}$ & Statistic & $P$ & $\begin{array}{c}\text { SCID } \\
\mathbf{N}=97\end{array}$ & $\begin{array}{l}\text { No SCID } \\
\mathrm{N}=415\end{array}$ & Statistic & $P$ \\
\hline MDQ Score & $9.92 \pm 2.12$ & $9.02 \pm 1.90$ & $t=2.503$ & $P=.751$ & $3.77 \pm 2.30$ & $4.15 \pm 2.76$ & $t=-1.232$ & $P=.169$ \\
\hline Age & $47.5 \pm 11.3$ & $48.1 \pm 11.9$ & $t=-0.318$ & $P=.751$ & $51.0 \pm 12.4$ & $52.2 \pm 13.7$ & $t=-0.637$ & $P=.524$ \\
\hline $\begin{array}{l}\text { Gender } \\
\quad \text { Female (\%) }\end{array}$ & 87.4 & 80.4 & $\chi^{2}=1.209$ & $P=.272$ & 86.0 & 79.7 & $\chi^{2}=1.983$ & $P=.159$ \\
\hline $\begin{array}{l}\text { Ethnicity } \\
\text { NHW (\%) } \\
\text { AA (\%) } \\
\text { HIS (\%) }\end{array}$ & $\begin{array}{r}77.0 \\
13.8 \\
2.3\end{array}$ & $\begin{array}{r}74.5 \\
17.6 \\
3.9\end{array}$ & $\chi^{2}=1.123$ & $P=.771$ & $\begin{array}{r}80.6 \\
11.8 \\
7.5\end{array}$ & $\begin{array}{l}68.4 \\
17.5 \\
10.8\end{array}$ & $x^{2}=6.978$ & $P=.073$ \\
\hline $\begin{array}{l}\text { Education } \\
\text { High school } \\
\text { graduate (\%) }\end{array}$ & 87.2 & 86.0 & $\chi^{2}=0.040$ & $P=.841$ & 89.1 & 83.0 & $\chi^{2}=2.110$ & $P=.146$ \\
\hline H/O BPD (\%) & 35.6 & 30.8 & $\chi^{2}=0.320$ & $P=.572$ & 12.0 & 11.1 & $\chi^{2}=0.057$ & $P=.811$ \\
\hline
\end{tabular}

NHW, non-Hispanic white; AA, African American; HIS, Hispanic; H/O BPD, history of bipolar disorder; MDQ, Mood Disorder Questionnaire; SCID, Structured Clinical Interview based on the DSM-IV.

bipolar NOS reported a previous diagnosis of bipolar disorder. Of the patients who did not report a history of bipolar disorder, $21.9 \%$ (30 of 137) were diagnosed as bipolar on the SCID: 17 bipolar I, 11 bipolar II, and 2 bipolar NOS. Thus, of the 180 patients taking antidepressants who completed the SCID interview, 29 (16.1\%) had a previous history of bipolar disorder confirmed, 31 (17.2\%) had a new diagnosis of bipolar disorder, and 14 (7.8\%) had a previous diagnosis that was not confirmed.

\section{Discussion}

In this study of patients in a primary care clinic who were receiving antidepressants for depression, we found that $21.3 \%$ screened positive for bipolar disorder. Approximately half of these people had never been diagnosed with bipolar disorder. Those screening positive were younger, more likely to be white, and more likely to be living alone. They were less likely to be married and less likely to be employed.

Table 4. Structured Clinical Interview Results versus MDQ Results

\begin{tabular}{lrrr}
\hline & \multicolumn{2}{c}{ SCID } & \\
\cline { 2 - 3 } & Positive & Negative & Total \\
\hline MDQ & & & \\
Positive & 45 & 41 & 86 \\
Negative & 14 & 80 & 94 \\
Total & 59 & 121 & 180 \\
\hline
\end{tabular}

These findings support the value of screening for bipolar disorder in depressed patients in primary care settings. Our study found that more than 1 in 5 patients who were receiving an antidepressant for depression screened positive for bipolar disorder. The majority (67\%) of these patients had never been diagnosed as bipolar. The likelihood that they were receiving mood stabilizers and other appropriate treatments for bipolar disorder was low.

A positive screen on the MDQ, however, is not a diagnosis of bipolar disorder. It indicates that a more thorough evaluation for bipolar disorder should be conducted. A good starting point in a primary care setting is to ask about the symptoms of bipolar disorder in the patient and the patient's family.

Because bipolar patients are more likely to seek treatment for their depressive states than for their

Table 5. Operating Characteristics of the MDQ Compared with Previous Reports

\begin{tabular}{lccc}
\hline & $\begin{array}{c}\text { Current } \\
\text { Study* }\end{array}$ & $\begin{array}{c}\text { Psychiatric } \\
\text { Outpatient }\end{array}$ & $\begin{array}{c}\text { General } \\
\text { Population }^{\ddagger}\end{array}$ \\
\hline Sensitivity & 0.580 & 0.73 & 0.281 \\
Specificity & 0.930 & 0.90 & 0.972 \\
\hline * Primary care outpatients treated for depression (adjusted for \\
sampling). \\
${ }^{\dagger}$ Psychiatric outpatient clinics focused on mood disorders. ${ }^{7}$ \\
${ }^{\ddagger}$ Adult general population randomly selected from a group of \\
respondents in a nationwide epidemiological general population \\
sample.
\end{tabular}


Table 6. Previous History of Bipolar Disorder by SCID Diagnosis

\begin{tabular}{lccr}
\hline & \multicolumn{3}{c}{$\begin{array}{c}\text { SCID Diagnosis of Bipolar } \\
\text { Disorder }\end{array}$} \\
\cline { 2 - 4 } & Positive & Negative & Total \\
\hline Previous history of & 29 & 14 & 43 \\
Bipolar disorder by & 30 & 107 & 137 \\
Self-report & 59 & 121 & 180 \\
\hline
\end{tabular}

manic or hypomanic states, clinicians should consider the possibility of bipolar disorder for all patients presenting for treatment of major depression. ${ }^{13}$ Although the clinical presentation of bipolar and unipolar depression may be identical, a history of mania or hypomania must be taken into consideration by the clinician when initiating a treatment plan. Treating a bipolar patient as a unipolar depressed patient may be a recipe for making a bad situation worse. Antidepressant treatment in bipolar disorder patients may only exacerbate the problem by inducing switching or rapid cycling. ${ }^{14,15}$

The imputed prevalence was similar to rates reported for other studies of depressed patients. To our knowledge, this is the first time anyone has looked specifically at patients being treated with antidepressants. Manning et $\mathrm{al}^{1}$ evaluated 108 consecutive anxious and/or depressed patients in a family practice setting with a semistructured interview. They found that more than one fourth of the patients had bipolar disorder, the majority of whom were bipolar II. The mean age of his sample was 34, 16 years younger than ours. The age difference may account for the higher prevalence he found. The patient population in the study described by Benazzi ${ }^{3}$ was similarly substantially younger than ours. He reported that $49 \%$ of consecutively evaluated depressed patients in a private outpatient psychiatric practice in Italy had bipolar disorder. The majority of those patients were bipolar II.

Based on the SCID interview, we found an adjusted sensitivity of 0.580 and a specificity of 0.930 . The sensitivity was slightly less than that found for unselected patients presenting to psychiatry mood disorder clinics. The specificity was somewhat higher. This may be due in part to the characteristics of the sample selected for the SCID that screened negative on the MDQ. We attempted to complete a SCID interview on all subjects who screened positive on the MDQ. However, resources did not allow us to screen all subjects who screened negative on the MDQ. We conducted SCID interviews on a limited sample of patients who were negative on the MDQ. On average, the subjects who were not diagnosed as bipolar from the SCID interview scored one point higher on the MDQ 13-item symptom checklist than subjects who were not selected to complete the SCID interview. In fact, $33.9 \%$ of patients without a diagnosis of bipolar disorder endorsed 7 or more symptoms compared with only $11.1 \%$ of patients who did not complete the SCID interview.

There are several limitations to this study. First, the UTMB clinic may not be representative of other primary care settings, and the results may not be generalizable. Second, the MDQ may be identifying conditions other than bipolar disorder, such as attention deficit hyperactivity disorder and anxiety disorders. We did not assess for these conditions in the SCID interview; therefore, we have no information to help address this issue.

Nevertheless, the study does suggest that a significant number of patients being treated with antidepressants in a primary care setting are likely to have bipolar disorder. This has important implications for clinical course and treatment.

\section{References}

1. Manning JS, Haykal RF, Connor PD, Akiskal HS. On the nature of depressive and anxious states in a family practice setting: the high prevalence of bipolar II and related disorders in a cohort followed longitudinally. Compr Psychiatry 1997;38:102-8.

2. Hantouche EG, Akiskal HS, Lancrenon S, et al. Systematic clinical methodology for validating bipolar-II disorder: data in mid-stream from a French national multi-site study (EPIDEP). J Affect Disord 1998;50:163-73.

3. Benazzi F. Prevalence of bipolar II disorder in outpatient depression: a 203-case study in private practice. J Affect Disord 1997;43:163-6.

4. Angst J. The emerging epidemiology of hypomania and bipolar II disorder. J Affect Disord 1998;50:14351.

5. Hirschfeld RMA, Vornik LA. Course and treatment of bipolar depression. In: Bowden CL, editor. Diagnosis and management of bipolar disorders. London (UK): Science Press; 2004. p. 28-40.

6. American Psychiatric Association. Diagnostic and statistical manual of mental disorders (DSM-IV), 4th ed. Washington (DC): American Psychiatric Association; 1994.

7. Hirschfeld RMA, Williams JB, Spitzer RL, et al. Development and validation of a screening instrument for 
bipolar spectrum disorder: the Mood Disorder Questionnaire. Am J Psychiatry 2000;157:1873-5.

8. Hirschfeld RMA, Holzer C, Calabrese JR, et al. Validity of the Mood Disorder Questionnaire: a general population study. Am J Psychiatry 2003;160: $178-80$.

9. First MB, Spitzer RL, Gibbon M, Williams JBW. Structured clinical interview for DSM-IV Axis I disorders, patient edition. SCID-I/P, Version 2.0. New York: New York State Psychiatric Institute, Biometrics Research; 1994.

10. Spitzer RL, Williams JB, Gibbon M, First MB. The structured clinical interview for DSM-III-R (SCID). I: History, rationale, and description. Arch Gen Psychiatry 1992;49:624-9.

11. Williams JB, Gibbon M, First MB, et al. The structured clinical interview for DSM-III-R (SCID). II.
Multisite test-retest reliability. Arch Gen Psychiatry 1992;49:630-6.

12. Cantor SB, Katten MW. Determining the area under the curve for a binary diagnostic test. Med Decic Making 2001;20:468-70.

13. Hirschfeld RMA. The Mood Disorder Questionnaire: a simple, patient-rated screening instrument for bipolar disorder. Prim Care Companion J Clin Psychiatry 2002;4:9-11.

14. Ghaemi SN, Rosenquist KJ, Ko JY, Baldassano CF, Kontos NJ, Baldessarini RJ. Antidepressant treatment in bipolar versus unipolar depression. Am J Psychiatry 2004;161:163-5.

15. Ghaemi SN, Hsu DJ, Soldani F, Goodwin FK. Antidepressants in bipolar disorder: the case for caution. Bipolar Disord 2003;5:421-33. 